\title{
NONCOMMUTATIVE DEFORMATION OF INSTANTONS AND VORTEXES
}

\author{
AKIFUMI SAKO
}

Communicated by Akira Yoshioka

\begin{abstract}
We study noncommutative (NC) instantons and vortexes. At first, we construct instanton solutions which are deformations of instanton solutions on commutative Euclidean four-space. We show that the instanton numbers of these $\mathrm{NC}$ instanton solutions coincide with the commutative solutions. Next, we also deform vortex solutions similarly and we show that their vortex numbers are unchanged under the NC deformation.
\end{abstract}

\section{Introduction}

Instanton connections in the four dimensional Yang-Mills theory are defined by

$$
F^{+}=\frac{1}{2}(1+*) F=0
$$

where $F$ is a curvature two-form and $*$ is the Hodge star operator.

The NC instanton solutions were constructed with the ADHM method in $[1,15]$. The ADHM construction which generate the instanton $\mathrm{U}(N)$ gauge field require a pair of the two complex vector spaces $V=\mathbb{C}^{k}, W=\mathbb{C}^{N}$. Here $k$ is an integer. Introduce $B_{1}, B_{2} \in \operatorname{Hom}(V, V), I \in \operatorname{Hom}(W, V)$ and $J \in \operatorname{Hom}(V, W)$ called ADHM data such that

$$
\begin{aligned}
\mu_{\mathbf{R}} & :=\left[B_{1}, B_{1}^{\dagger}\right]+\left[B_{2}, B_{2}^{\dagger}\right]+I I^{\dagger}-J^{\dagger} J=\zeta \operatorname{Id}_{k} \\
\mu_{\mathbf{C}} & :=\left[B_{1}, B_{2}\right]+I J=0 .
\end{aligned}
$$

Here $\zeta$ is a NC parameter and its detail will appear in the following. Using these ADHM data we can construct NC instanton and call it NC ADHM instanton in the following. NC ADHM instantons and deformed instantons from the commutative ADHM construction is unknown.

It was also shown that instanton numbers of NC ADHM instantons do not depend on the NC parameter $[4,5,9,16,17]$. These results may imply that the instanton 
numbers in Euclidean four-space are invariant under NC deformation. Furthermore topological charges might be preserved under the NC deformation for any other solitons in gauge theories in Euclidean spaces. These problems are main themes of this article.

In this paper, we construct a $\mathrm{NC}$ formal instanton solution which is a deformation of the commutative instanton. We show that the NC instanton number for this NC instanton is independent of $\hbar$ (Theorem 4). This result supports our conjecture on the independence of the $\mathrm{NC}$ instanton number for $\mathrm{NC} \mathbb{R}^{4}$. In addition, we also construct a $\mathrm{NC}$ vortex solution which is a deformation of commutative vortex solution and showed that its vortex number is undeformed [11,12].

\section{Notations}

NC Euclidean $2 n$-space is given by the following commutation relations:

$$
\left[x^{\mu}, x^{\nu}\right]_{\star}=x^{\mu} \star x^{\nu}-x^{\nu} \star x^{\mu}=\mathrm{i} \theta^{\mu \nu}, \quad \mu, \nu=1,2, \ldots, 2 n
$$

where $\left(\theta^{\mu \nu}\right)$ is a real, $x$-independent, skew-symmetric matrix, called the matrix of NC parameters. The $\star$ multiplication is known as the Moyal product [14]. The Moyal product (or star product) is defined on functions by

$$
f(x) \star g(x)=f(x) g(x)+\sum_{n=1}^{\infty} \frac{1}{n !} f(x)\left(\frac{\mathrm{i}}{2} \overleftarrow{\partial}_{\mu} \theta^{\mu \nu} \vec{\partial}_{\nu}\right)^{n} g(x)
$$

Here $\overleftarrow{\partial}_{\mu}$ and $\vec{\partial}_{\nu}$ are partial derivatives with respect to $x^{\mu}$ for $f(x)$ and to $x^{\nu}$ for $g(x)$, respectively.

The curvature two form $F$ is defined by

$$
F:=\frac{1}{2} F_{\mu \nu} \mathrm{d} x^{\mu} \wedge \star \mathrm{d} x^{\nu}=\mathrm{d} A+A \wedge \star A
$$

where $\wedge \star$ is defined by

$$
A \wedge \star A:=\frac{1}{2}\left(A_{\mu} \star A_{\nu}\right) \mathrm{d} x^{\mu} \wedge \mathrm{d} x^{\nu} .
$$

To consider smooth NC deformations, we introduce a parameter $\hbar$ and a fixed constant $\theta_{0}^{\mu \nu}<\infty$ with

$$
\theta^{\mu \nu}=\hbar \theta_{0}^{\mu \nu}
$$

We define the commutative limit by letting $\hbar \rightarrow 0$. 


\section{NC Deformation of Instantons}

In this section, we consider the Yang-Mills theory on the $\mathrm{NC} \mathbb{R}^{4}$. Instanton solutions satisfy the (NC) instanton equation $F^{+}=\frac{1}{2}(1+*) F=0$. Formally we expand the connection as

$$
A_{\mu}=\sum_{l=0}^{\infty} A_{\mu}^{(l)} \hbar^{l} .
$$

Then

$$
\begin{aligned}
A_{\mu} \star A_{\nu} & =\sum_{l, m, n=0}^{\infty} \hbar^{l+m+n} \frac{1}{l !} A_{\mu}^{(m)}(\overleftrightarrow{\Delta})^{l} A_{\mu}^{(n)} \\
\overleftrightarrow{\Delta} & \equiv \frac{i \overleftarrow{\partial}_{\mu} \theta_{0}^{\mu \nu} \vec{\partial}_{\nu} .}{}
\end{aligned}
$$

We introduce the selfdual projection operator $P$ by

$$
P:=\frac{1+*}{2}, \quad P_{\mu \nu, \rho \tau}=\frac{1}{2}\left(\delta_{\mu \rho} \delta_{\nu \tau}-\delta_{\nu \rho} \delta_{\mu \tau}+\epsilon_{\mu \nu \rho \tau}\right) .
$$

Then the instanton equation is written as

$$
P_{\mu \nu, \rho \tau} F^{\rho \tau}=0 \text {. }
$$

In the NC case, the $l$-th order equation of (11) is given by

$$
\begin{aligned}
& P^{\mu \nu, \rho \tau}\left(\partial_{\rho} A_{\tau}^{(l)}-\partial_{\tau} A_{\rho}^{(l)}+\mathrm{i}\left[A_{\rho}^{(l)}, A_{\tau}^{(l)}\right]+C_{\rho \tau}^{(l)}\right)=0 \\
& C_{\rho \tau}^{(l)}:=\sum_{(p ; m, n) \in I(l)} \hbar^{p+m+n} \frac{1}{p !}\left(A_{\rho}^{(m)}(\overleftrightarrow{\Delta})^{p} A_{\tau}^{(n)}-A_{\tau}^{(m)}(\overleftrightarrow{\Delta})^{p} A_{\rho}^{(n)}\right)(12) \\
& I(l) \equiv\left\{(p ; m, n) \in \mathbb{Z}^{3} \mid p+m+n=l, p, m, n \geq 0, m \neq l, n \neq l\right\} .
\end{aligned}
$$

Note that $C^{(l)}$ is a given function when we solve these equations recursively. The 0 -th order equation is the commutative instanton equation with solution $A_{\mu}^{(0)}$ a commutative instanton. The asymptotic behavior of commutative instanton $A_{\mu}^{(0)}$ is given by

$$
A^{(0)}=g \mathrm{~d} g^{-1}+O\left(|x|^{-2}\right), \quad g \mathrm{~d} g^{-1}=O\left(|x|^{-1}\right)
$$

where $g \in G$ and $G$ is a gauge group. We introduce covariant derivatives associated to the commutative instanton connection by

$$
D_{\mu}^{(0)} f:=\partial_{\mu} f+\mathrm{i}\left[A_{\mu}^{(0)}, f\right], \quad D_{A^{(0)}} f:=\mathrm{d} f+A^{(0)} \wedge f .
$$


Using this, (12) is given by

$$
P^{\mu \nu, \rho \tau}\left(D_{\rho}^{(0)} A_{\tau}^{(l)}-D_{\tau}^{(0)} A_{\rho}^{(l)}+C_{\rho \tau}^{(l)}\right)=0 .
$$

In the following, we fix a commutative anti-selfdual connection $A^{(0)}$. We impose the following gauge fixing condition for $A^{(l)}(l \geq 1)$

$$
A-A^{(0)}=D_{A^{(0)}}^{*} B, \quad B \in \Omega_{+}^{2}
$$

where $D_{A^{(0)}}^{*}$ is defined by

$$
\left(D_{A^{(0)}}^{*}\right)_{\rho}^{\mu \nu} B_{\mu \nu}=\delta_{\rho}^{\nu} D^{(0) \mu} B_{\mu \nu}-\delta_{\rho}^{\mu} D^{(0) \nu} B_{\mu \nu} .
$$

We expand $B$ in $\hbar$ as we did with $A$. Then $A^{(l)}=D_{A^{(0)}}^{*} B^{(l)}$. In this gauge, the equation (15) is given by

$$
P D_{A^{(0)}} D_{A^{(0)}}^{*} B^{(l)}+P C^{(l)}=0 .
$$

Using the fact that the $A^{(0)}$ is an anti-selfdual connection, (18) simplifies to

$$
2 D_{(0)}^{2} B^{(l) \mu \nu}+P^{\mu \nu, \rho \tau} C_{\rho \tau}^{(l)}=0
$$

where

$$
D_{(0)}^{2} \equiv D_{A^{(0)}}^{\rho} D_{A^{(0)} \rho} .
$$

We derive some properties of the Green's function of $D_{(0)}^{2}$. (We restrict ourselves to $\mathrm{U}(n)$ gauge theory.)

We consider the Green's function for $D_{(0)}^{2}$

$$
D_{(0)}^{2} G_{0}(x, y)=\delta(x-y)
$$

where $\delta(x-y)$ is a four dimensional delta function. Instantons in commutative $\mathbb{R}^{4}$ are given by the ADHM construction [1], and arbitrary commutative instantons are in one-to-one correspondence with ADHM data. $G_{0}(x, y)$ has been constructed in [2] :

$$
G_{0}(x, y)=\frac{\left[v_{1}(x) \otimes v_{2}(x)\right]^{\dagger}(1-\mathfrak{M})\left[v_{1}(y) \otimes v_{2}(y)\right]}{4 \pi^{2}(x-y)^{2}} .
$$

Here $\mathfrak{M}$ and $v_{1}, v_{2}$ are determined by the ADHM data and $v_{i}$ is a bounded function. Using this Green's function, we solve the equation (19) as

$$
B^{(l) \mu \nu}=-\frac{1}{2} \int_{\mathbb{R}^{4}} G_{0}(x, y) P^{\mu \nu, \rho \tau} C_{\rho \tau}^{(l)}(y) \mathrm{d}^{4} y
$$


and the NC instanton $A=\sum A^{(l)}$ is given by $A^{(l)}=D_{A^{(0)}}^{*} B^{(l)}$. The key fact used in the following proposition is that the asymptotic behavior of Green's function of $D_{(0)}^{2}$ is given by

$$
G_{0}(x, y)=O\left(|x-y|^{-2}\right), \quad|x-y|>>1 .
$$

To list some features of Green's functions like $G_{0}$, see some propositions.

Proposition 1. Let $G(x, y)$ be a Green's function on $\mathbb{R}^{4}$ written as

$$
G(x, y)=\frac{b(x, y)}{|x-y|^{2}}
$$

where $b(x, y)$ is a bounded function. Let $f(x)$ be a function such that $|f(x)|<$ $\frac{C}{1+|x|^{4}}$ where $C$ is some constant. We define $F(x)$ by

$$
F(x):=\int_{\mathbb{R}^{4}} G(x, y) f(y) \mathrm{d}^{4} y .
$$

Then $F(x)=O\left(|x|^{-2}\right)$.

We gave a proof of this proposition in [12].

We introduce the notation $O^{\prime}\left(|x|^{-m}\right)$ as in [3]. If $s$ is a function of $\mathbb{R}^{4}$ which is $O\left(|x|^{-m}\right)$ as $|x| \rightarrow \infty$ and $\left|D_{(0)}^{k} s\right|=O\left(|x|^{-m-k}\right)$, then we denote this natural growth condition by $s \in O^{\prime}\left(|x|^{-m}\right)$.

Examining the proof of Proposition 1, and keeping track of estimates for higher derivatives, we have the following (see Lemma 3.3.36 in [3]).

Proposition 2. If $f(x) \in O\left(|x|^{-m}\right)$ and $\left|D_{(0)}^{2} f(x)\right|=O^{\prime}\left(|x|^{-m-2}\right)$, then $f(x) \in$ $O^{\prime}\left(|x|^{-m}\right)$.

We apply these propositions to our case, then we get the following theorem.

Theorem 3. If $C^{(l)} \in O^{\prime}\left(|x|^{-4}\right)$, then $\left|B^{(k)}\right|<O^{\prime}\left(|x|^{-2}\right)$.

The proof is given in [12]. In our case, $C_{\rho \tau}^{(1)}=O^{\prime}\left(x^{-4}\right)$ by (13), and so $\left|B^{(1)}\right|<$ $O^{\prime}\left(|x|^{-2}\right)$ from Theorem 3 and $\left|A^{(1)}\right|<O^{\prime}\left(|x|^{-3}\right)$ as $A^{(l)}=D_{A^{(0)}}^{*} B^{(l)}$. Repeating the argument $l$ times, we get

$$
\left|A^{(l)}\right|<O^{\prime}\left(|x|^{-3+\epsilon}\right), \quad \text { for any } \epsilon>0 .
$$


Now is the time to estimate the instanton number. The instanton number is defined by

$$
I_{\hbar}:=\frac{1}{8 \pi^{2}} \int \operatorname{tr} F \wedge \star F
$$

We rewrite (26) as

$$
\frac{1}{8 \pi^{2}} \int \operatorname{tr} \mathrm{d}\left(A \wedge \star \mathrm{d} A+\frac{2}{3} A \wedge \star A \wedge \star A+\right)+\frac{1}{8 \pi^{2}} \int \operatorname{tr} P_{\star}
$$

where $P_{\star}$

$$
\frac{1}{3}\{F \wedge \star A \wedge \star A+2 A \wedge \star F \wedge \star A+A \wedge \star A \wedge \star F+A \wedge \star A \wedge \star A \wedge \star A\} .
$$

The term $\int \operatorname{tr} P_{\star}$ is 0 in the commutative limit, but does not vanish in NC space. The cyclic symmetry of trace is broken by the NC deformation.

The terms in $\int \operatorname{tr} P_{\star}$ are typically written as

$$
\int_{\mathbb{R}^{d}} \operatorname{tr}\left(P \wedge \star Q-(-1)^{n(4-n)} Q \wedge \star P\right)
$$

where $P$ and $Q$ be an $n$-form and a $(4-n)$-form $(n=0, \ldots, 4)$, respectively, and let $P \wedge Q$ be $O\left(\hbar^{k}\right)$. The lowest order term in $\hbar$ vanishes because of the cyclic symmetry of the trace, i.e., $\int \operatorname{tr}\left(P \wedge Q-(-1)^{n(4-n)} Q \wedge P\right)=0$. The term of order $\hbar$ is given by

$$
\begin{aligned}
& \frac{\mathrm{i}}{2} \int_{\mathbb{R}^{4}} \operatorname{tr}\left\{\hbar \theta_{0}^{\mu \nu}\left(\partial_{\mu} P \wedge \partial_{\nu} Q\right)\right\} \\
& \quad=\frac{\mathrm{i}}{2} \int_{\mathbb{R}^{4}}(n !(4-n) !) \epsilon^{\mu_{1} \mu_{2} \mu_{3} \mu_{4}} \operatorname{tr} \mathrm{d}\left\{(* \theta) \wedge\left(P_{\mu_{1} \ldots \mu_{n}} \mathrm{~d} Q_{\mu_{n+1} \ldots \mu_{4}}\right)\right\}
\end{aligned}
$$

where $* \theta=\epsilon_{\mu \nu \rho \tau} \theta^{\rho \tau} \mathrm{d} x^{\mu} \wedge \mathrm{d} x^{\nu} / 4$. These integrals are zero if $P_{\mu_{1} \ldots \mu_{n}} \mathrm{~d} Q_{\mu_{n+1} \ldots \mu_{4}}$ is $O^{\prime}\left(|x|^{-(4-1+\epsilon))}(\epsilon>0)\right.$. Similarly, higher order terms in $\hbar$ in (29) can be written as total divergences and hence vanish under the decay hypothesis. This fact and (25) imply that $\int \operatorname{tr} P_{\star}=0$.

From the above discussion and (25), we get following theorems.

Theorem 4. Let $A_{\mu}^{(0)}$ be a commutative instanton solution in $\mathbb{R}^{4}$ given by the ADHM construction. There exists a formal NC instanton solution $A_{\mu}=\sum_{l=0}^{\infty} A_{\mu}^{(l)} \hbar^{l}$ 
such that the instanton number $I_{\hbar}$ defined by (26) is independent of the NC parameter $\hbar$, i.e.,

$$
\frac{1}{8 \pi^{2}} \int \operatorname{tr} F \wedge \star F=\frac{1}{8 \pi^{2}} \int \operatorname{tr} F^{(0)} \wedge F^{(0)} .
$$

\section{NC Vortex}

In this section we study NC deformation of the vortex solutions. We consider the Abelian-Higgs model in commutative $\mathbb{R}^{2}$ and deform vortex solutions into $\mathrm{NC}$ vortexes.

\subsection{Commutative Vortex Solutions}

At first, we consider vortexes on commutative $\mathbb{R}^{2}$ with a Higgs field $\phi$, a complex scalar field. Let $G$ be the group of gauge transformations associated to $\mathrm{U}(1)$. For $g \in G$, the gauge transformation is defined as $\phi \rightarrow g \phi$. We introduce the covariant derivative by $\nabla_{\mu} \phi=\partial_{\mu} \phi-\mathrm{i} A_{\mu} \phi$.

For later convenience, we introduce complex coordinates for $\mathbb{R}^{2}$ and $A_{\mu}$. $z=$ $\left(x^{1}+\mathrm{i} x^{2}\right) / \sqrt{2}, \bar{z}=\left(x^{1}-\mathrm{i} x^{2}\right) / \sqrt{2}$, and define differential operators $\partial, \bar{\partial}$ by $\partial=\left(\partial_{1}-\mathrm{i} \partial_{2}\right) / \sqrt{2}, \bar{\partial}=\left(\partial_{1}+\mathrm{i} \partial_{2}\right) / \sqrt{2}$, and define complex gauge fields by $A=\left(A_{1}-\mathrm{i} A_{2}\right) / \sqrt{2}, \bar{A}=\left(A_{1}+\mathrm{i} A_{2}\right) / \sqrt{2}$. The gauge transformations are $A \rightarrow \mathrm{i} g \partial g^{-1}+A, \quad \bar{A} \rightarrow-\mathrm{i} \bar{\partial} g g^{-1}+\bar{A}$. The curvature is expressed in the coordinates $z, \bar{z}$ as

$$
F_{z z}=F_{\bar{z} \bar{z}}=0, \quad F_{z \bar{z}}=\mathrm{i} F_{12}=\partial \bar{A}-\bar{\partial} A .
$$

We define the magnetic field $B$ by $B:=-\mathrm{i} F_{z \bar{z}}$.

The vortex equations are given by

$$
\bar{D} \phi=(\bar{\partial}-\mathrm{i} \bar{A}) \phi=0, \quad B+\phi \bar{\phi}-1=0 .
$$

Solutions of these equations (32) minimize the energy functional. We list some facts concerning vortex solutions.

Theorem 5 (Taubes [10]) Let $\left(A_{0}, \phi_{0}\right)$ be a smooth solution of (32). The vortex number,

$$
N_{0}:=\frac{1}{2 \pi} \int_{\mathbb{R}^{2}} \mathrm{~d}^{2} x B_{0}
$$

is an integer equal to the winding number of $\lim _{|z| \rightarrow \infty} \phi_{0}$, where $B_{0}:=B\left(A_{0}\right)$. 
There is a useful formula.

$$
0<\frac{1}{2}\left(1-\left|\phi_{0}(x)\right|^{2}\right)<M(\epsilon) \mathrm{e}^{-r(1-\epsilon)}
$$

where $r=|x|$. From (34), the asymptotic behaviors of the $\left(A_{0}, \phi_{0}\right)$ for large radius $r$ are given by

$$
\left|\phi_{0}\right| \sim 1-C \mathrm{e}^{-r(1-\epsilon)}, \quad\left|\partial \phi_{0}\right| \sim\left|\bar{\partial} \phi_{0}\right| \sim \frac{C^{\prime}}{r}, \quad\left|A_{0}\right| \sim \frac{C^{\prime \prime}}{r} .
$$

Here, $C, C^{\prime}, C^{\prime \prime}$ are some constants.

In the following, we investigate the $\mathrm{NC}$ deformations of this theory. In particular, we will carefully discuss whether the vortex number is constant.

\subsection{The NC Abelian Higgs Model}

At first, let coordinates of NC Euclidean space $\mathbb{R}_{\theta}^{2}$ be $x^{\mu}, \mu=1,2$, with commutation relations

$$
\left[x^{\mu}, x^{\nu}\right]=\mathrm{i} \hbar \epsilon^{\mu \nu}, \quad \mu, \nu=1,2
$$

where $\epsilon^{\mu \nu}=-\epsilon^{\nu \mu},\left(\epsilon^{12}=1\right)$ and $\hbar$ is a NC parameter.

The curvature components of the connection $A$ are given by

$$
F_{z z}=F_{\bar{z} \bar{z}}=0, \quad F_{z \bar{z}}=\mathrm{i} F_{12}=\partial_{z} A_{\bar{z}}-\partial_{\bar{z}} A_{z}-\mathrm{i}\left[A_{z}, A_{\bar{z}}\right]_{\star}
$$

where $[A, B]_{\star}:=A \star B-B \star A$. The magnetic field is defined by $B:=-\mathrm{i} F_{z \bar{z}}$. The NC vortex equations are defined by

$$
\bar{D} \star \phi=(\bar{\partial}-\mathrm{i} \bar{A}) \star \phi=0, \quad B+\phi \star \bar{\phi}-1=0 .
$$

We call solutions of these equations NC vortices or NC vortex solutions.

The formal expansions of the fields are

$$
\phi=\sum_{n=0}^{\infty} \hbar^{n} \phi_{n}(z, \bar{z}), \quad A=\sum_{n=0}^{\infty} \hbar^{n} A_{n}(z, \bar{z}) .
$$

The $k$-th order equations for (37) are

$$
\begin{aligned}
-\mathrm{i}\left(\partial \bar{A}_{k}+\bar{\partial} A_{k}\right)+\phi_{k} \bar{\phi}_{0}+\phi_{0} \bar{\phi}_{k}-\delta_{k 0}+C_{k}(z, \bar{z}) & =0 \\
\bar{\partial} \phi_{k}-\mathrm{i} \bar{A}_{k} \phi_{0}-\mathrm{i} \bar{A}_{0} \phi_{k}+D_{k}(z, \bar{z}) & =0 .
\end{aligned}
$$

Here $C_{k}(z, \bar{z})$ is the coefficient of $\hbar^{k}$ in $-[A, \bar{A}]_{\star}+\phi \star \bar{\phi}-\left(\phi_{k} \bar{\phi}_{0}+\phi_{0} \bar{\phi}_{k}\right)$. Similarly, $D_{k}(z, \bar{z})$ is the coefficient of $\hbar^{k}$ in $-\mathrm{i} \bar{A} \star \phi-\left(-\mathrm{i} \bar{A}_{k} \phi_{0}-\mathrm{i} \bar{A}_{0} \phi_{k}\right)$. 
Therefore when we solve these equations recursively, then $C_{k}$ and $D_{k}$ are given functions.

In particular in the case of $k=0,(39)$ and (40) coincide with the commutative $\mathrm{U}(1)$ vortex equations (32), i.e., $\bar{D} \phi_{0}=\left(\bar{\partial}-\mathrm{i} \bar{A}_{0}\right) \phi_{0}=0$ and $B_{0}+\phi_{0} \bar{\phi}_{0}-1=0$, where $B_{0}=-\mathrm{i}\left(\partial \bar{A}_{0}-\bar{\partial} A_{0}\right)$.

Setting

$$
\varphi_{k}:=\frac{\phi_{k}}{\phi_{0}}+\frac{\bar{\phi}_{k}}{\bar{\phi}_{0}}=2 \operatorname{Re}\left(\frac{\phi_{k}}{\phi_{0}}\right) \text { and } d_{k}=\frac{D_{k}}{\phi_{0}}
$$

(40) and (39) are simplified as

$$
\left(-\Delta+\left|\phi_{0}\right|^{2}\right) \varphi_{k}=E_{k}
$$

Here $\Delta=\partial \bar{\partial}$ and

$$
E_{k}:=-C_{k}+\partial d_{k}-\bar{\partial} \bar{d}_{k} .
$$

From (34), there exists a positive constant $C$ such that

$$
\left|D_{1}\right|<\frac{C}{1+r^{3}}, \quad\left|C_{1}\right|<\frac{C}{1+r^{4}}, \quad\left|E_{1}\right|<\frac{C}{1+r^{4}} .
$$

We use (44) to prove some of our main theorems.

\subsection{NC Vortex Number}

In this section, we show that the vortex number is constant for vortex solutions that are given by NC deformations of Taubes' vortex solutions.

\section{The Schrödinger Equation and Vortex Solutions}

To show that there exists a unique NC vortex solution deformed from the Taubes' vortex solution, we consider the stationary Schrödinger equation

$$
(-\Delta+V(x)) u(x)=f(x)
$$

in $\mathbb{R}^{2}$, where $V(x)$ is a real valued $C^{\infty}$ function such that

(a1) $V(x) \geq 0$, for any $x \subset \mathbb{R}^{2}$

(a2) There exist $K \subset \mathbb{R}^{2}$ and there exists $c>0$ such that $K$ is a compact set and for $x \in \mathbb{R}^{2} \backslash K, V(x) \geq c$

(a3) There exist $x_{1}, \ldots, x_{N} \in \mathbb{R}^{2}$ such that $V\left(x_{i}\right)=0, V(x)>0$ for $x \notin\left\{x_{1}, \ldots, x_{N}\right\}$

(a4) For any $\alpha=\left(\alpha_{1}, \alpha_{2}\right) \in \mathbb{Z}_{+}^{2}$, There exists a positive constant $C_{\alpha}$ such that $\left|\partial_{x}^{\alpha}(V-c)\right| \leq C_{\alpha}$ for any $x \in \mathbb{R}^{2}$. 
We note that the system (42) satisfies the assumptions $(a 1)-(a 4)$. We set

$$
H_{l}(n):=\left\{f ;|| f \|:=\sup _{x \in \mathbb{R}^{2}}\left(1+|x|^{n}\right)\left|\partial_{x}^{\alpha} f(x)\right|<\infty \text { for any }|\alpha| \leq l\right\}
$$

for $n \in \mathbb{Z}_{+}$. We let $C, C_{\alpha}$, etc. denote unimportant positive constants whose value may change from line to line unless otherwise stated. The next theorem's proof was given by using standard techniques of Green's function [11].

Theorem 6. There exists a unique solution $u \in H_{l}(n)$ of (45) for any $f \in H_{l}(n)$.

These theorems imply the following main theorem.

Theorem 7. Let $A_{0}$ and $\phi_{0}$ be a Taubes' vortex solution stated in section 4.1, in other words, $\left(A_{0}, \phi_{0}\right)$ satisfy the equations (32) with the condition (34). Then there exists a unique solution $(A, \phi)$ of the $N C$ vortex equations (37) with $\left.A\right|_{\theta=0}=$ $A_{0},\left.\phi\right|_{\theta=0}=\phi_{0}$, and its vortex number is preserved:

$$
N=N_{0}, \quad \frac{1}{2 \pi} \int_{\mathbb{R}^{2}} \mathrm{~d}^{2} x B=\frac{1}{2 \pi} \int_{\mathbb{R}^{2}} \mathrm{~d}^{2} x B_{0} .
$$

Outline of the Proof. Consider (45) with $V(x)=\left|\phi_{0}\right|^{2}$ and $f(x)=E_{k}$. From the facts in section 4.1, we find $V(x)$ satisfies $(a 1)-(a 4)$. Next, we consider $E_{k}$. From (44), $E_{1} \in H_{\infty}$ (4). If $E_{i} \in H_{\infty}(2 i+2)(i=1, \ldots, k-1)$, as a result of Theorem 6 , there exist unique solutions $\varphi_{1}, \ldots, \varphi_{k-1}$. Then we find $E_{k} \in H_{\infty}(2 k+2)$. Therefore $E_{k} \in H_{\infty}(2 k+2)$ is proved for arbitrary $k$. Theorem 6 is applicable to (42) for arbitrary $k$, then it is shown that each $\varphi_{k}$ is determined uniquely. Finally, using the asymptotic behavior of $\phi_{0}$, (44) and so on, we can get the result $N=N_{0}$.

\section{Conjectures and Open Problems}

We conjecture instanton numbers in Euclidean four-space are invariant under NC deformations. In this article we introduce a gauge condition (16), so this conjecture is not proved for general instantons. On the other hand, for Taubes' vortexes, we proved that vortex number is invariant under NC deformations. Furthermore, it is natural to expect that topological charges are preserved under NC deformations for any other solitons in gauge theories in Euclidean spaces.

From these observations, a new question arises: "How to distinguish instantons (solitons) that preserve their instanton number (topological charges) under NC 
deformation?" From the proofs in $[11,12]$ we can find that a hint has already appeared. The hint is the volume of the space is $\infty$, in the proofs to show the topological charges are not deformed. Therefore it is natural to expect that instanton number depends on the NC parameter in a finite volume NC space.

Here is an example [6-8, 13]. An instanton solution on $T^{4}$ for $U\left(N^{2}\right)$ gauge theory is given by

$$
\begin{array}{ll}
D_{1}=\partial_{1}, & D_{2}=\partial_{2}+\frac{1}{2} \frac{k}{N}\left(x_{1} \mathbf{1}_{N}\right) \otimes \mathbf{1}_{N} \\
D_{3}=\partial_{3}, & D_{4}=\partial_{4}-\frac{1}{2} \frac{k}{N}\left(x_{3} \mathbf{1}_{N}\right) \otimes \mathbf{1}_{N} .
\end{array}
$$

The instanton number is given by $k^{2}$. After NC deformation, the instanton number is also deformed to

$$
\frac{1}{8 \pi^{2}} \int_{T^{4}} \operatorname{tr} F \wedge \star F=\frac{k^{2} N^{2}}{(N-k \hbar)^{2}}
$$

and it depends on the NC parameter. Anyway, it is left as an open problem to find the way to distinguish instantons whose instanton numbers do not depend on NC parameter.

\section{Acknowledgement}

The author acknowledges the support by KAKENHI 20740049 Grant-in-Aid for Young Scientists (B).

\section{References}

[1] Atiyah M., Hitchin N., Drinfeld V. and Manin Yu., Construction of Instantons, Phys. Lett. A 65 (1978) 185-187.

[2] Corrigan E., Goddard P. and Templeton S., Instanton Green's Functions and Tensor Products, Nucl. Phys. B 151 (1979) 93-124 .

[3] Donaldson S. and Kronheimer P., The Geometry of Four-Manifolds, Oxford Univ. Press, Oxford, 1990.

[4] Furuuchi K., Instantons on Noncommutative $\mathbb{R}^{4}$ and Projection Operators, Prog. Theor. Phys. 103 (2000) 1043-1068, hep-th/ 9912047.

[5] Furuuchi K., Topological Charge of U(1) Instantons, hep-th/0010006. 
[6] Ganor O., Ramgoolam S. and Taylor W., Branes, Fluxes and Duality in M(atrix)-theory, Nucl. Phys. B 492 (1997) 191-204, hep-th/ 9611202.

[7] Hamanaka M. and Kajiura H., Gauge Fields on Tori and T-duality, Phys. Lett. B 551 (2003) 360-368, hep-th/ 0208059.

[8] Ho P., Twisted Bundle on Quantum Torus and BPS States in Matrix Theory, Phys. Lett. B 434 (1998) 41-47, hep-th/ 9803166.

[9] Ishikawa T., Kuroki S. and Sako A., Instanton Number on Noncommutative $\mathbb{R}^{4}$, hep-th/0201196. Calculation of the Pontrjagin Class for U(1) Instantons on Noncommutative $\mathbb{R}^{4}$ JHEP 0208, 028 (2002).

[10] Jaffe A. and Taubes C., Vortices and Monopoles, Birkhäuser, Boston, 1980.

[11] Maeda Y. and Sako A., Are Vortex Numbers Preserved?, J. Geom. Phys. 58 (2008) 967-978, math-ph/0612041.

[12] Maeda Y. and Sako A., Noncommutative Deformation of Instantons, J. Geom. Phys. 58 (2008) 1784-1791, arXiv: 0805.3373

[13] Morariu B. and Zumino B., Super Yang-Mills on The Noncommutative Torus, hep-th/9807198.

[14] Moyal J., Quantum Mechanics as a Statistical Theory, Proc. Cambridge Phil. Soc. 45 (1949) 99-124.

[15] Nekrasov N. and Schwarz A., Instantons on Noncommutative $\mathbb{R}^{4}$ and $(2,0)$ Superconformal Six Dimensional Theory, Commun. Math. Phys. 198 (1998) 689-703, hep-th/9802068.

[16] Sako A., Instanton Number of Noncommutative $U(N)$ Gauge Theory, JHEP 0304 (2003) 023, hep-th/ 0209139.

[17] Tian Y., Zhu C. and Song X., Topological Charge of Noncommutative ADHM Instanton, hep-th/0211225.

Akifumi Sako

Department of General Education

Kushiro National College of Technology

Otanoshike-Nishi 2-32-1, Kushiro 084-0916

JAPAN

E-mail address: sako@kushiro-ct.ac.jp 